\title{
A vanishing callosal lesion on brain imaging in a patient with meningoencephalitis
}

\author{
Thashi Chang ${ }^{1}$, RadikaKarunarathne ${ }^{2}$, Chandima De $\mathrm{Mel}^{2}$ \\ ${ }^{1}$ Department of Clinical Medicine, University of Colombo, Kynsey Road, Colombo 08. Sri Lanka. \\ ${ }^{2}$ Nawaloka Hospitals PLC, Colombo 02, Sri Lanka.
}

\begin{abstract}
Case vignette
A 21-year-old previously healthy man had become progressively unresponsive five days after developing highgrade fever. On examination, he was unconscious (GCS 3/15), with equally reacting normal-sized pupils and normal optic fundi, and marked neck stiffness. His blood investigations showed neutrophil leucocytosis (total white cells $21,250 / \mu 1 ; 88 \%$ neutrophils) and serum sodium of $112 \mathrm{mmol} / \mathrm{l}$. Magnetic resonance imaging of the brain was normal except for a non-enhancing focal hyperintensity on T2 and FLAIR sequences with diffusion restriction and low signal on ADC map in the splenium of the corpus callosum (Figure). Cerebrospinal fluid analysis showed polymorphocytic pleocytosis (225 cells $/ \mu 1 ; 80 \%$ polymorphocytes), elevated protein (99 $\mathrm{mg} / \mathrm{dl}$ ) and a CSF: plasma glucose ratio of 0.28 . A diagnosis of pyogenic meningoencephalitis was established. The patient made a complete recovery after a 14-day course of intravenous meropenem and vancomycin. MRI repeated 14 days after completion of antibiotics showed complete resolution of the splenial hyperintensity.
\end{abstract}

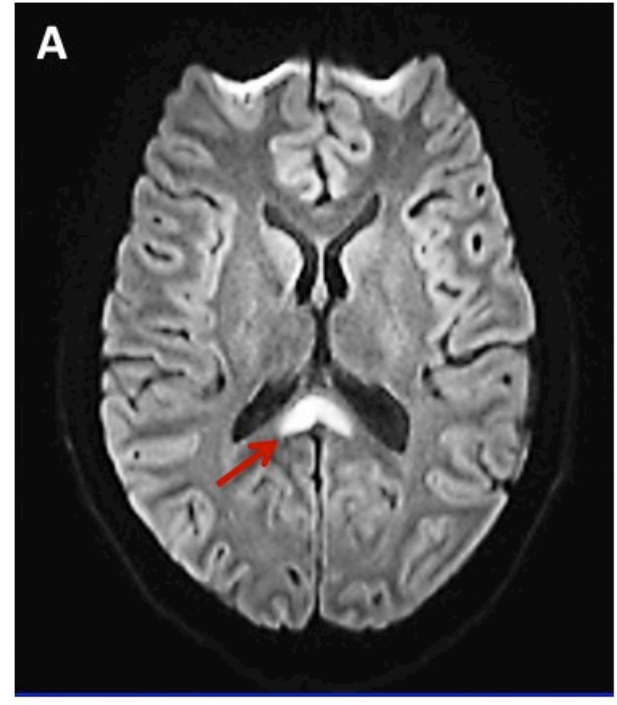

MRIFLAIR image

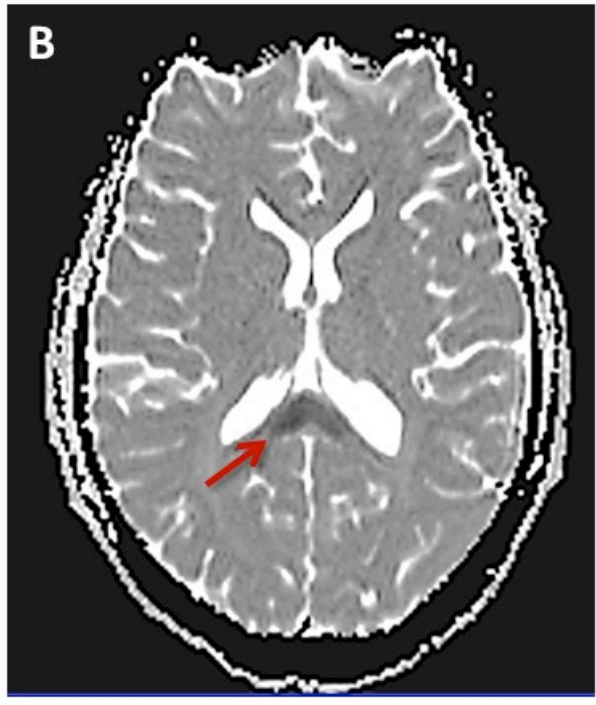

MRI ADC map

\section{Q:What is the radiological diagnosis?}

Author responsible for correspondence:

Prof. Thashi Chang

Professor in Neurology

Department of Clinical Medicine

Faculty of Medicine, Colombo.

Email: thashichang@gmal.com

https://orcid.org/0000-0002-6027-7883

\section{Article Information}

Key words: splenium, corpus callosum, meningoencephalitis

Date of submission:18.12.2017

Date of acceptance: 31.12.2017

DOI:

http://doi.org/10.4038/cjms.v54i2.4910 


\section{A:Reversible splenial lesion syndrome}

The corpus callosum is a bundle of commissural fibres that connects the left and right cerebral hemispheres. The posterior end of the corpus callosum is known as the splenium. Lesions in the corpus callosum and the splenium have been associated with distinct neurological disorders. Reversible splenial lesion syndrome (RESLES) is one such disorder.

RESLES as observed in our patient, is a rare clinicoradiological syndrome described in association with antiepileptic drug withdrawal, cerebral infection, high-altitude cerebral oedema (HACE), or metabolic disorders (hypoglycemia and hypernatremia) [1]. Rarely it has been reported in association with systemic lupus erythomatosus, anorexia $\mathrm{B}_{12}$ deficiency and hereditary motor and sensory neuropathy.

\section{References}

1.Garcia-Monco JC, Cortina IE, Ferreira E, Martinez A, Ruiz L, Cabrera A, et al. Reversible splenial lesion syndrome (RESLES): what's in a name? Journal of neuroimaging : official journal of the American Society of Neuroimaging. 2011;21(2):e1-14. nervosa, vitamin

The pathogenesis of RESLES is mostly attributed to cytotoxic oedema with the exception of HACE in which vasogenic oedema is thought to be the underlying mechanism. The reason for the predilection for the splenium of the corpus callosum remains obscure, but a relative lack of adrenergic tone and failure of autoregulation at this site is one hypothesised mechanism [2]. No callosal disconnection syndromes have been described in RESLES while complete clinical and radiological resolution with time is usual [1]. It usually carries a good prognosis except in those patients with an underlying severe disorder.

The recognition of this distinct radiological appearance should prompt appropriate differential diagnosis and reassurance of a generally good prognosis.

2.Hackett PH, Yarnell PR, Hill R, Reynard K, Heit J, McCormick J. High-altitude cerebral edema evaluated with magnetic resonance imaging: clinical correlation and pathophysiology. JAMA. 1998;280(22):1920-5. 